\title{
Image De-noising by Bayesian Regression
}

\author{
Shimon Cohen and Rami Ben-Ari \\ Orbotech Ltd., Yavneh, Israel \\ \{Shimon.Cohen, Rami.Ben-Ari\}@orbotech.com \\ http://www.orbotech.co.il/
}

\begin{abstract}
We present a kernel based approach for image de-noising in the spatial domain. The crux of evaluation for the kernel weights is addressed by a Bayesian regression. This approach introduces an adaptive filter, well preserving edges and thin structures in the image. The hyper-parameters in the model as well as the predictive distribution functions are estimated through an efficient iterative scheme. We evaluate our method on common test images, contaminated by white Gaussian noise. Qualitative results show the capability of our method to smooth out the noise while preserving the edges and fine texture. Quantitative comparison with the celebrated total variation (TV) and several wavelet methods ranks our approach among state-of-the-art denoising algorithms. Further advantages of our method include the capability of direct and simple integration of the noise PDF into the de-noising framework. The suggested method is fully automatic and can equally be applied to other regression problems.
\end{abstract}

Keywords: Image de-noising, Bayesian regression, Adaptive filtering.

\section{Introduction}

The need for efficient image restoration methods has grown with the massive production of digital images and movies of all kinds, often taken in poor conditions. No matter how good cameras are, an image improvement is always desirable to extend their range of action. The valid challenge of denoising methods is removing the noise without creating artifacts, while preserving the image edges and fine structures. Such denoising attempts are referred as adaptive filtering.

Many methods have been suggested in the past for image adaptive denoising. One class of methods filters the image via the frequency domain using wavelets [174612. Although yielding excellent results in terms of PSNR, yet these methods often produce particular visual artifacts such as ringing.

Another approach performs the filtering in the spatial domain, known as steerable filters. In the domain of PDE and diffusion methods the inhomogeneous (i.e. shift variant yet isotropic) and anisotropic diffusion approaches gained popularity for their desired properties on edge preserving [91113. In this framework the so called Total Variation regularization (TV) attracted special attention 9513. These PDE based approaches among other non-linear methods can be approximated by a kernel based filtering where the kernel is shift variant [15. However, 
in these methods the adjustment of the model to the noise distribution is highly non-trivial and practically hidden under the distance measure (norm) used in the model. Furthermore, these methods often need a tuning parameter, adjusted by the user, in order to obtain adequate performance.

Non-deterministic modeling approaches 2716] rely on the data for adaptively varying the kernel. Our method belongs to this latter approach, where the noise PDF is an explicit part of our statistical model. The key ingredient of our approach is the use of simple but efficient Bayesian estimation model. Bayesian estimation seeks a predictive probability distribution function (PPDF), i.e prediction is done through model averaging. We view the image denoising as a regression problem in the spirit shown by Tipping [10]. While in [10] a Taylor expansion (to the second degree) is used to obtain an exact solution, we avoid this approximation by utilizing an efficient iterative procedure for solution of the regression problem.

Often measurement models are considered to be linear in the unknown image. Yet, more successful methods have taken a nonlinear estimation approach to this inverse problem [2 16]. In this work, we perform our regression on a transformed domain by Radial Basis Functions (RBF) [3] and impose a prior on the RBF's weights in order to avoid over fitting.

Experiments show that the proposed approach can reduce noise from corrupted images while preserving edge components efficiently. Despite the simplicity of our method both in its concept and implementation, the denoising results are among the best reported in the literature. It is further executed without any parameter setting or user intervention. The suggested regression scheme is not limited to image de-noising and can be employed for other regression problems.

\section{Problem Formulation}

Usually in a data-model matching procedure, one tries to optimize the likelihood of the data, i.e, the probability of the data given the model $p(D \mid M)$. We assume the model has parameters (a.k.a weights) arranged in the vector $\mathbf{w}$ and consider the method of Maximum a-posterior probability (MAP) with automatic inference of the regularization parameters. We then deduce the probability of the model given the data, i.e. $p(M \mid D)$ and use it to construct the predictive distribution.

\subsection{Bayesian Regression}

In this work, we assume that image pixels are corrupted by additive white Gaussian noise with an unknown variance $\sigma^{2}$ :

$$
y_{i}-t_{i} \sim N\left(0, \sigma^{2}\right)
$$

where, $t_{i}$ denotes the targets namely, the observed intensities and $y_{i}$ the output of a regression method. The likelihood of the data under identically independent distribution (i.i.d) is given by: 


$$
p(D \mid M)=\prod_{i=1}^{K} \frac{1}{\sqrt{2 \pi} \sigma} \exp \left(-\frac{\left(y_{i}-t_{i}\right)^{2}}{2 \sigma^{2}}\right)
$$

where $K$ denotes the kernel size. The first stage of the restoration is based on regression of a pixel value based on the data in a kernel. To this end often linear regression model is employed. However, a linear model imposes a severe limitation on the allowed relation between the pixels in the kernel. We therefore consider a set of non-linear functions $\phi_{i}(x)$ mapping the input vector (observations) to a new space, allowing a more flexible model:

$$
y(\mathbf{x} ; \mathbf{w})=\sum_{i=1}^{K} w_{i} \phi_{i}(\mathbf{x})+w_{0}=\mathbf{w}^{T} \phi(\mathbf{x})+w_{0}
$$

where $\mathbf{w}:=\left[w_{1}, w_{2}, \ldots, w_{K}\right]^{T}$ is the parameter vector of the model to estimate in a certain kernel and $K$ denotes the kernel size (number of training samples). We hereby describe a new Bayesian probabilistic approach for learning $p(M \mid D)$, where $D$ in this case presents the corrupted image. As the basis functions $\phi(\mathbf{x}):=$ $\left[\phi_{1}(\mathbf{x}), \phi_{2}(\mathbf{x}), \ldots, \phi_{K}(\mathbf{x})\right]$ we choose the following $\mathrm{RBF}$ :

$$
\phi_{i}(x):=\exp \left(-\frac{\left\|x-t_{i}\right\|^{2}}{2 r^{2}}\right) .
$$

In the context of image de-noising, we define a regressor imposed on a training sample set at the size of the kernel. For a kernel size $k \times k$ the regressor will therefore have a training sample size of $K=k^{2}$. The width of the Gaussian functions, $r$, depends on the size $k$. We set this width to be $r=k / 2.5$. Note that the regression (3) produces a model for prediction of the intensities for all the pixels in the kernel domain.

Estimation of the weights (model) is the crux of the proposed denoising algorithm. It is well known that that maximum likelihood estimation of $\mathbf{w}$ and $\sigma^{2}$ from (3) will lead to severe over-fitting [1]. To avoid this, we impose an additional constraint on the parameters, through the addition of "complexity" penalty term to the likelihood or error function. Here, though, we adopt a Bayesian perspective, and "constrain" the parameters to obey a prior probability distribution. The preference for smoother (less complex) functions is made by the popular choice of zero mean Gaussian prior distribution over the weights w. Note that when $\mathbf{w}=0$, then $w_{0}$ will obtain the mean intensity value in the kernel. We also apply a prior on $w_{0}$ as normal distribution around the mean value $m$, i.e. $w_{0} \sim N\left(m, \sigma_{0}^{2}\right)$ and define the corresponding precision parameter as $\alpha_{0}=1 / \sigma_{0}^{2}$. Assuming conditional independence in $\mathbf{w}$ components and characterization by individual precisions $\alpha_{k}=1 / \sigma_{k}^{2}$, namely hyper-parameters (with corresponding std $\sigma_{k}$ ), yields the following prior probability function:

$$
p(M)=\frac{1}{(2 \pi)^{K}} \prod_{i=1}^{K} \alpha_{i}^{1 / 2} \exp \left(-\frac{\alpha_{i} w_{i}^{2}}{2}\right) \cdot \alpha_{0}^{1 / 2} \exp \left(-\frac{\alpha_{0}\left(w_{0}-m\right)^{2}}{2}\right)
$$


Importantly, there is an individual hyper-parameter associated with every weight. This mechanism moderates the strength of the prior in space, adaptively.

Having defined the prior, Bayesian inference proceeds by computing, from Bayes' rule, the posterior over all unknowns given the data. To this end, we wish to optimize the posterior probability for model parameters $M$ given the data $D$ :

$$
M^{*}=\arg \max _{M} p(M \mid D)=\arg \max _{M} p(D \mid M) p(M)
$$

This condition is equivalent to maximization of the corresponding log term substituting Eq. (2) and Eq. (5) in Eq. (6), ignoring the constants:

$$
\begin{aligned}
L\left(\mathbf{w}, \boldsymbol{\alpha}, w_{0}, \alpha_{0}, \sigma\right)=-K \ln (\sigma)-\sum_{i=1}^{K} \frac{\left(y_{i}-t_{i}\right)^{2}}{2 \sigma^{2}} & -\sum_{i=1}^{K} w_{i}^{2} \alpha_{i} / 2+\sum_{i=1}^{K} 1 / 2 \ln \left(\alpha_{i}\right) \\
& -\frac{\left(w_{0}-m\right)^{2}}{2} \alpha_{0}+\frac{1}{2} \ln \left(\alpha_{0}\right) .
\end{aligned}
$$

The optimal model is then a result of maximization of (7). Note that when a certain point in the kernel obtains a high mismatch value (e.g. $\frac{\left(y_{i}-t_{i}\right)^{2}}{2 \sigma^{2}} \gg 1$ ), then the associated weight will reduce significantly due to the maximization process. This effectively "switches off" the influence of the corresponding input in regression, as desired. Nevertheless, in areas where the image is nearly constant (in sense of local mean), a limited data discrepancy is expected and maximization of (7) yields weights with homogeneous distribution (i.e. $\mathbf{w} \rightarrow 0$, and $w_{0} \neq 0$ ). This is a highly effective mechanism for preserving the image structure in the restoration process while filtering out the noise, and it is a direct by product of our Bayesian formulation. Note that the suggested regression scheme doesn't make any use of spatial relations between points. Therefore this model is not limited to images and can be used for other regression problems.

Maximization of $L$ is pendent to the following necessary conditions:

$$
\begin{aligned}
\frac{\partial L}{\partial w_{k}} & =-\frac{\sum_{i=1}^{K}\left(y_{i}-t_{i}\right) \phi_{k}\left(t_{i}\right)}{\sigma^{2}}-w_{k} \alpha_{k} \\
\frac{\partial L}{\partial w_{0}} & =-\frac{\sum_{i=1}^{K}\left(y_{i}-t_{i}\right)}{\sigma^{2}}-\left(w_{0}-m\right) \alpha_{0} \\
\frac{\partial L}{\partial \alpha_{i}} & =\frac{-w_{i}^{2}}{2}+\frac{1}{2 \alpha_{i}}=0 \\
\frac{\partial L}{\partial \alpha_{0}} & =\frac{1}{2 \alpha_{0}}-\frac{\left(w_{0}-m\right)^{2}}{2}=0
\end{aligned}
$$

As for estimation of the noise std $\sigma$ we use the none biased estimate as follows:

$$
\sigma^{2}=\frac{\sum_{i=1}^{K}\left(y_{i}-t_{i}\right)^{2}}{K-1}
$$


The unknown values of $w_{i}, \alpha_{i}, w_{0}, \alpha_{0}, \sigma$ which satisfy Eq. (8, 12) cannot be obtained in a closed form, and hereby we suggest an iterative scheme for their estimation. Each iteration step is composed of three stages. The conjugate gradient [8] scheme is used for estimation of $w_{k}$ in Eq.(8). This step is followed by evaluation of $w_{0}$ in (9) and the precision parameters $\alpha_{i}$ and $\alpha_{0}$ in Eq. (10[11). The next iteration step is then applied after updating the noise std in (12). At each phase only one set of variables are updated to satisfy the equation, while the others are kept constant. The algorithm flow is then:

\section{Algorithm Flow:}

1) Initialization

2) Estimate w from Eq.(8).

3) Estimate $w_{0}$ from Eq.(9).

4) Update precision values $\alpha_{k}$ and $\alpha_{0}$ using Eqs.(10) and (11).

5) Update noise std evaluation $\sigma$ by Eq.(12).

6) Repeat steps 1-5 until convergence.

We experienced convergence of the scheme in just two iterations. The above model now can be used to produce a set of hypotheses for Bayesian inference as described in the following section.

\subsection{Bayesian Inference}

In this work we consider a set of $\mathcal{K}$ hypotheses for each data point, governed from the kernel centralized on the considered point and another $\mathcal{K}-1$ overlapping kernels. We seek to compute the expected value $E(\hat{t} \mid \hat{x})$ associated with a new test point $\hat{x}$ mapped to the target $\hat{t}$. In Bayesian framework this is conducted by calculation of the predictive distribution [1]:

$$
E(\hat{t} \mid \hat{x})=\int \hat{t} \cdot p(\hat{t}, \mathfrak{m} \mid \hat{x}, D) d \mathfrak{m}=\int \hat{t} \cdot p(\hat{t} \mid \mathfrak{m}, \hat{x}, D) p(\mathfrak{m} \mid D) d \mathfrak{m}
$$

where $\mathfrak{m}$ presents the model, in this case the regressor outcome. Evaluation $p(\mathfrak{m} \mid D)$ is based on computation of the marginal probability (see (6-6) ). Since the the posterior $p(\hat{t} \mid \mathfrak{m}, \hat{x}, D)$ can not be directly computed we use the following decomposition [110]:

$$
p(\hat{t} \mid \mathfrak{m}, \hat{x}, D)=\int p(\hat{t}, w \mid \hat{x}, \mathfrak{m}, D) d w=\int p(\hat{t} \mid w, \hat{x}, \mathfrak{m}) \cdot p(w \mid \mathfrak{m}, D) d w
$$

The term $p(\hat{t} \mid w, \hat{x}, \mathfrak{m})$ has a normal distribution where the distance is measured by absolute difference in intensity values. As for $p(w \mid \mathfrak{m}, D)$, the distribution is 
also normal having the posterior mean obtained from Eq. 18 as its mode. Thus the expression under the integral sign in (14) presents convolution of two Gaussian distributions resulting in a new Gaussian characterized by the parameters of the distributions it was composed of 1 . Note however that our prediction is based on several hypotheses, obtained here from neighbouring kernels. This paradigm is inferred from Bias-Variance decomposition concept. Since the error is composed from bias and variance, Bayesian model averaging significantly improves the prediction by coping in fact, with the notorious variance part of the error.

\section{Results}

In this section, we will demonstrate the performance of the proposed approach on popular test images, contaminated with white Gaussian noise. Our data set incorporates images with different characteristics, from piecewise smooth to highly textured images with fine structures. Figure 1 shows our test bed comprised of the celebrated "Barbara", "Lena", "Boat" and "Pepper" images all $512 \times 512$ in size. For sake of illustration, the insets were corrupted with noise having standard deviation of 10, 20 and 30 grey levels. Figure 2 shows the restoration results for the Barbara and Pepper test cases with two different kernel sizes and noise level of $30 \mathrm{std}$. The high quality of restorations in both of these disparate cases, the piecewise smooth Pepper image and the fine textured Barbara, demonstrate the high capability of the proposed approach. Despite the excessive noise level, the denoised images are visually appealing. There are slight artifacts in the Barbara result when restored with a small kernel size of $3 \times 3$. This effect is vanished when the kernel is enlarged to $5 \times 5$, but yields a slightly lower PSNR. The larger kernel size produces visually improved denoising in both cases and higher PSNR for the Pepper image having a subtle texture. One can observe carefully the restoration of the delicate texture pattern in Barbara image, shown in the
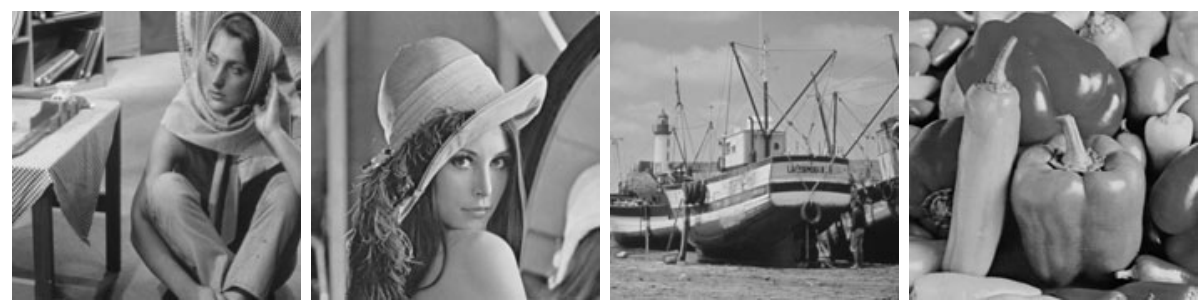

Fig. 1. The test data set prior to noise contamination. From left to right: Barbara, Lena, Boat and Peppers.

${ }^{1}$ The interested reader is referred to [1] for details in the derivation. 


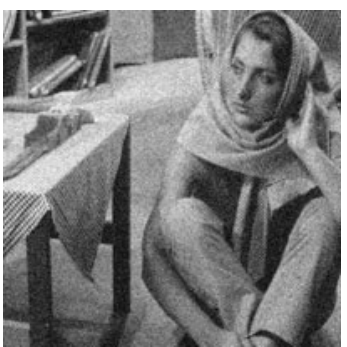

PSNR

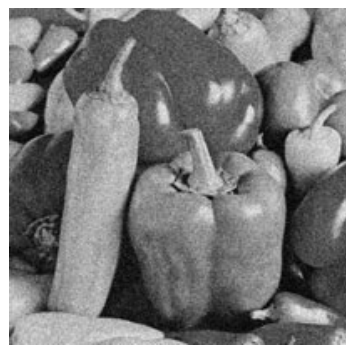

PSNR

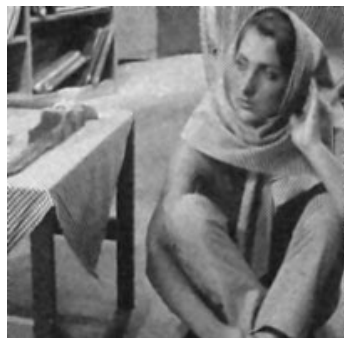

27.65

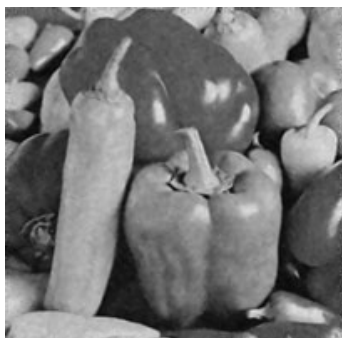

28.23

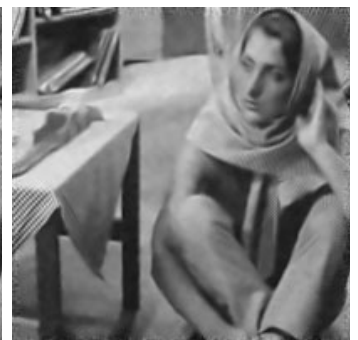

27.24

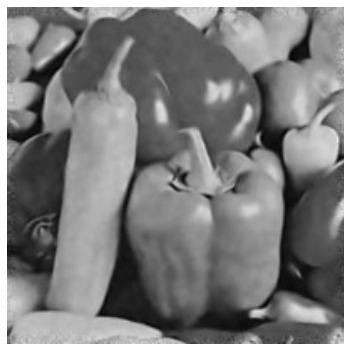

29.23

Fig. 2. Image denoising results for Barbara (top) and Pepper (down) images. Left: Image corrupted with 30 std Gaussian noise. Middle: Restored image with kernel size $3 \times 3$. Right: Restored image with kernel size $5 \times 5$. subfigures indicate PSNR values in $\mathrm{dB}$.
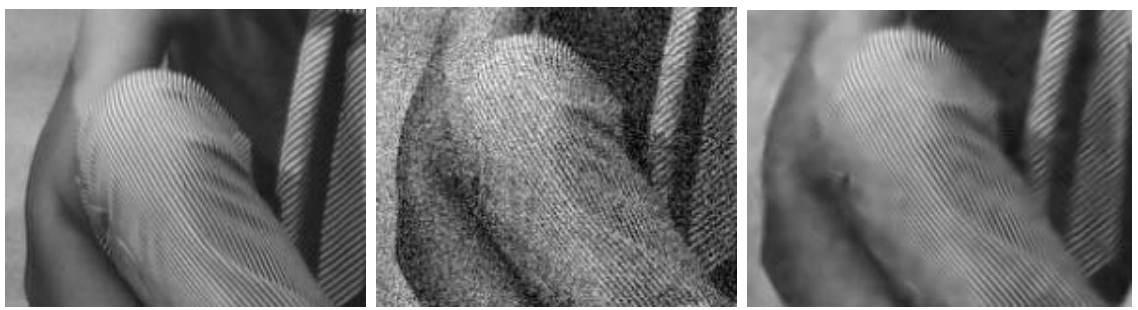

Fig. 3. Barbara zoomed in. Left: Original patch. Middle: Input image with 30 std noise. Right: Restoration result with the proposed method, kernel size 3.

cropped patches in Figure 3. We further evaluate our method quantitatively by the common PSNR measure:

$$
P S N R=20 \log _{10}\left(\frac{255}{\sqrt{\text { Mean Square Error }}}\right) .
$$


For comparison we present results from 5 methods in the literature, comprised of two popular and three recently published methods based on wavelet transforms. The PSNR values of the restored images are listed in Table 12 . From this table it is clear that published algorithms [12 1417] all substantially outperform the TV and the hard-threshold (HT) methods, with the MVM presenting the best results. The suggested Bayesian method shows comparable results and performs superiorly under high noise levels.

Table 1. Comparison of performance for image denoising using different algorithms in terms of PSNR. Methods: TV: Total Variation minimization in variational approach 13, HT: Hard Threshold, BS: Bivariate Shrinkage [14, GSM: Gaussian Scale Mixtures [12, MVM: Multivariate Statistical Model [17, Bayes.: Our Bayesian approach with $3 \times 3$ kernel size. Best results are in bold. Note: HT, GSM and MVM act in wavelet transform domain.

\begin{tabular}{l|ccc|ccc|ccc|ccc}
\hline Method & \multicolumn{3}{|c}{ Barbara } & \multicolumn{3}{c}{ Lena } & \multicolumn{3}{c}{ Boat } & \multicolumn{3}{c}{ Pepper } \\
\hline & 10 & 20 & 30 & 10 & 20 & 30 & 10 & 20 & 30 & 10 & 20 & 30 \\
\hline TV & 30.28 & 26.54 & 24.60 & 33.00 & 30.26 & 28.35 & 32.30 & 29.23 & 27.36 & 32.24 & 29.84 & 28.05 \\
HT & 31.99 & 27.68 & 25.43 & 34.48 & 31.29 & 29.31 & 32.63 & 29.31 & 27.42 & 33.59 & 29.73 & 27.55 \\
BS & 32.73 & 28.73 & 26.51 & 34.51 & 31.38 & 29.54 & 32.62 & 29.30 & 27.48 & 32.95 & 29.21 & 27.01 \\
GSM & 33.11 & 29.18 & 27.02 & 34.85 & 31.65 & 29.82 & 33.02 & 29.62 & 27.78 & 33.16 & 29.50 & 27.40 \\
MVM & $\mathbf{3 3 . 2 5}$ & 29.40 & 27.23 & $\mathbf{3 4 . 9 5}$ & $\mathbf{3 1 . 8 3}$ & $\mathbf{2 9 . 9 7}$ & $\mathbf{3 3 . 0 1}$ & $\mathbf{2 9 . 7 5}$ & 27.84 & 33.20 & 29.47 & 27.32 \\
Bayes. & 32.70 & $\mathbf{2 9 . 6 7}$ & $\mathbf{2 7 . 6 5}$ & 34.35 & 31.52 & 29.71 & 32.90 & 29.71 & $\mathbf{2 7 . 9 8}$ & $\mathbf{3 3 . 3 7}$ & $\mathbf{3 1 . 1 1}$ & $\mathbf{2 8 . 2 3}$ \\
\hline
\end{tabular}

The complexity of our method is dominated by the kernel size and the scheme used for approaching the solution of the linear system (8] 11). Considering the Conjugate Gradient method used the complexity of our scheme is $O(K \cdot n)$ when $K$ indicates the size of the kernel and $n$ the image size.

\section{Summary}

We hereby present a Bayesian approach for image de-noising in the spatial domain. The proposed method is based on kernel estimation, while the kernel weights are evaluated by regression using radial basis functions allowing a more flexible data modeling. The kernel weights are imposed with a prior probability distribution with individual hyper-parameters, moderating adaptively the strength of the prior (regularization), in space. The model unknowns are then evaluated efficiently through an iterative procedure. Finally, the intensity values

${ }^{2}$ Results for HT, GSM and MVM correspond to orthonormal wavelet transform as reported in [17]. 
are restored by Bayesian inference over hypotheses obtained from neighboring kernels.

The suggested method was illustrated on 4 popular test images contaminated with white additive Gaussian noise. Comparison to several recently published methods show comparative results, and introduces superior performance in the presence of large noise levels. Another advantage of the proposed approach is in the capability to explicitly incorporate the noise PDF in the model. It is therefore able to cope with non-Gaussian noise distributions e.g, a Poissonian PDF model obtained in SPECT medical images.

Finally, the proposed Bayesian approach is simple, generic, free of adjusting parameters and can be used in other regression based applications.

\section{References}

1. Bishop, C.M.: Pattern Recognition and Machine Learning. Springer Science+Business Media, LLC (2006)

2. Buades, A., Coll, B., Morel, J.M.: A review of image denoising methods, with a new one. Multiscale Model. Simul. 4(2), 490-530 (2005)

3. Buhman, M.D.: Radial Basis Functions, Theory and Implementations. Cambridge University Press, Cambridge (2003)

4. Chang, S.G., Vetterli, M.: Adaptive wavelet thresholding for image denoising and compression. IEEE Transaction on image processing 9(9), 1532-1546 (2000)

5. Combettes, P.L., Pesquet, J.C.: Image restoration subject to a total variation constraint. IEEE Trans. on Image Processing 13(9), 1213-1222 (2010)

6. Donoho, D.L.: De-noising by soft thresholding. IEEE Transactions on Information Theory 41(3), 613-627 (1995)

7. Halder, A., Shekhar, S., Kant, S., Mubarki, M., Pandey, A.: A new efficient adaptive spatial filter for image enhancement. In: Proc. Int. Conference on Computer Engineering and Applications, vol. 1

8. Hestenes, M., Stiefel, E.: Methods of conjugate gradients for solving linear systems. Journal of Research of the National Bureau of Standards 49(6), 409-436 (1952)

9. Loeza, C.B., Chen, K.: On high-order denoising models and fast algorithms for vector valued images. IEEE Transactions on Image Processing 19(6), 1518-1527 (2010)

10. Michael, T., Alex, S.: Sparse bayesian learning and relevance vector machine. IEEE Journal of Machine Learning research 1, 211-244 (2001)

11. Perona, P., Malik, J.: Scale space and edge detection using anisotropic diffusion. International Journal of Computer Vision 12(7), 629-639 (1990)

12. Portilla, J., Strela, V., Wainwright, M.J., Simoncelli, E.P.: Image denoising using scale mixtures of gaussians in the wavelet domain. IEEE Trans. on Image Processing 12(11), 1338-1351 (2003)

13. Rudin, L.I., Osher, S., Fetami, E.: None-linear total varaition based noise removal algorithms. Physica. D: Non-linear Phenomena 60, 259-268 (1992) 
14. Sendur, L., Selesnick, I.W.: Bivariate shrinkage with local variance estimation. IEEE Signal Processing Letters 9, 428-441 (2002)

15. Spira, A., Kimmel, R., Sochen, N.A.: A short- time beltrami kernel for smoothing images and manifolds. IEEE Transactions on Image Processing 16(6), 1628-1636 (2007)

16. Takeda, H., Farsiu, S., Milanfar, P.: Kernel regression for image processing and reconstruction. IEEE Transaction on Image Processing 16(2), 349-366 (2007)

17. Tan, S., Jiao, L.: Multivariate statistical models for image denoising in the wavelet domain. IEEE Transaction on Pattern Analysis and Machine Intelligence 75(2), 209-230 (2007) 